\title{
Transformer Oil Ageing Detection using Mach-Zender Interferometry Configuration as a Sensor
}

\author{
A. Razzaq, H. Zainuddin, F. Hanaffi, Y.Ying, Radhi M. Chyad, HA Razak
}

\begin{abstract}
Paper In this paper, the fabrication and characterisation of an optical fibre sensor for transformer oil ageing detection are presented. Bare fibre is used in the Mach-Zender interferometry (MZI) configuration as a sensing arm. Hydrofluoric acid (30\%) and a power meter are used in sensor fabrication. The MZI sensor is affected by the changes of the refractive index (RI) of the transformer oil. The sensor operates according to different output power levels at the receiving end of the optical sensor. Results agree with the AC breakdown voltage test and oil absorption spectrum test. This work contributes to the improvement of transformer oil monitoring systems by ensuring the availability of oil information and protecting such systems from damage.
\end{abstract}

Keywords : Transformer oil, Ageing measurement, Mach-Zender interferometry, Bare fibre, Chemical etching

\section{INTRODUCTION}

$\mathrm{P}$ ower system networks consist of three main parts namely, generation, transmission and distribution. Each of this part involves the use of high voltage equipment, such as power transformers, circuit breakers, bus bars and instrument transformers. Power transformer is a vital equipment in power system networks i.e. in the transmission and distribution sectors. Ensuring system reliability requires a stable operation for power transformers [1]. The insulation of transformers is typically insulating oil and paper, whereas transformer oils

Revised Manuscript Received on July 22, 2019.

A. Razzaq, Research Laboratory of High Voltage Engineering, Centre for Robotics and Industrial Automation (CeRIA), Faculty of Electrical Engineering, Universiti Teknikal Malaysia Melaka, Melaka, Malaysia. Email: alaautem9000@gmail.com

H. Zainuddin, Research Laboratory of High Voltage Engineering, Centre for Robotics and Industrial Automation (CeRIA), Faculty of Electrical Engineering, Universiti Teknikal Malaysia Melaka, Melaka, Malaysia. Email: hidayat@utem.edu.my

F. Hanaffi, Research Laboratory of High Voltage Engineering, Centre for Robotics and Industrial Automation (CeRIA), Faculty of Electrical Engineering, Universiti Teknikal Malaysia Melaka, Melaka, Malaysia. Email: farhan@utem.edu.my

Y.Ying, Research Laboratory of High Voltage Engineering, Centre for Robotics and Industrial Automation (CeRIA), Faculty of Electrical Engineering, Universiti Teknikal Malaysia Melaka, Melaka, Malaysia. Email: yingyi_95@hotmail.com

Radhi M. Chyad, Laser \& Optoelectronics Research Center, Directorate of Materials Research, Ministry of Science \& Technology, Baghdad, IRAQ. Email: chyad.qi64@gmail.com

HA Razak, Centre for Telecommunication Research and Innovation (CeTRI), Universiti Teknikal Malaysia Melaka (UTeM),. Email: hanim@utem.edu.my function as an insulator and a coolant. These oils are exposed to deterioration due to different factors, such as electrical and thermal stresses [2]. Contamination, excessive temperature and oxidation are the principal sources of oil degradation [3].

The ageing and degradation of oils increase the risk of catastrophic losses for transformers. Various tests are carried out to check the quality of transformer oils, and they include tests of viscosity, turbidity, moisture, breakdown voltage, acidity, etc. [4] [5]. Such tests are conducted by taking a sample from a transformer and sending it to laboratories to generate results that could aid in decision making. The breakdown test is the main test used to identify the level of quality and ageing of transformer oils. This type of test is affected by moisture, acidity and conducting material of the oil; its results are equivalent to oil quality [6].

In recent years, optical fibre sensors have been widely utilised in various fields, including environmental, chemical, biological and food industries, because of their unique properties, such as immunity to electromagnetic interference, good sensitivity, light weight and compact size. The quality of oils has been measured by different authors on the basis of different techniques, such as fibre Bragg gratings (FBG) [7], UV-visible spectrophotometry [8], Mach-Zender interferometry (MZI) [9], surface plasmon resonance [10] and NIR method [11] [12].

Since the last decade, an increase focus has been directed toward intensity modulation sensors. In reference [13], the water content of the transformer oil was measured by using a bare and bent multimode fibre as an optical sensor. The author explained how the output power intensity changes due to variations in the refractive index (RI) of transformer oils. Laskar et al. [14] presented a bare fibre for the real-time moisture measurement of transformer oils. The author used a polymer optical fibre sensor on the basis of the changing intensity of output power. A novel FBG-based intensity-modulated optical fibre sensor for partial discharge is presented in reference [15], this sensor can detect partial discharge from the high-frequency acoustic waves emitted from it.

Fibre optic interferometry configurations come in four types, namely, Fabry-Perot, Mach-Zender, Michelson and Sagnac. Given the design simplicity, flexibility and high sensitivity of MZI [16] [17], the MZI configuration is widely used in different sensing applications as a sensor, particularly in the form of a temperature sensor [18], RI sensor [19] and strain sensor [20]. 
The MZI configuration consists of two arms, that is, a sensing arm and a reference arm, as shown in Fig. 1. The input light is divided into two arms by using an optical coupler, and another coupler is used for light recombination. The reference arm is not exposed to variations, whereas it is vice versa for the sensing arm. The propagated light in the optical fibre suffers from leaks as an evanescent field waves when cladding

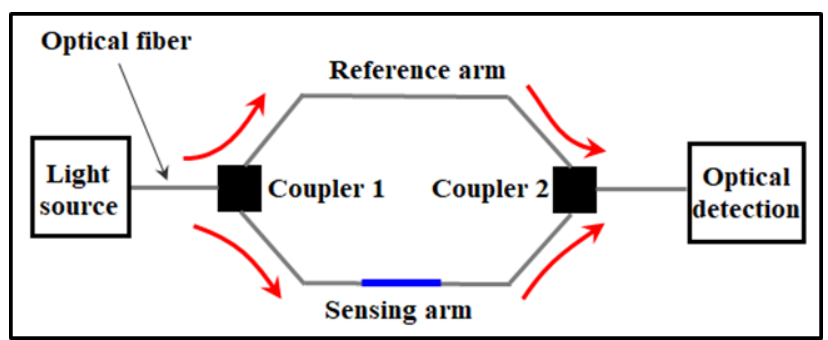

is removed; these properties increase the sensitivity of optical sensors to the surrounding media [21].

Fig. 1. Block diagram of MZI

In this paper, tapered fibres are used as a sensing arm in the MZI configuration to detect the ageing of transformer oils on the basis of the changing RI. This type of fibre is tapered using hydrofluoric acid (HF, 30\%) in accordance with the chemical etching method. The results are compared with those of a standard AC breakdown voltage test and oil absorption test for validation.

\section{PRINCIPLES OF TAPERED OPTICAL FIBRE SENSOR}

Light signals are propagated in the optical fibre wave guide in accordance with the theory of total internal reflection. The reflections are due to the differences between the RI of the core and the cladding of the optical fibre. When the cladding is tapered by reducing its diameter, light penetrates the fibre wall as an evanescent field. The evanescent field is affected by the surrounding medium. The surrounding medium is transformer oil, which functions as a new cladding. Tapering the fibre changes two parameters, namely, the number of light reflections and the incident angle. The reflection is increased when the incident angle reaches the critical angle $(\phi c)$. The sensitivity of the optical fibre is affected by the changes in the tapered ratio $(T R)$, as in reference [22].

$$
T R=\frac{P}{P_{0}}
$$

Where $T R$ is the tapered ratio, $P$ is the waist in $\mu \mathrm{m}$ and $P_{O}$ is the core radius in $\mu \mathrm{m}$. Factors such as core RI, cladding RI and external RI affect the light propagated inside fibres. The evanescent field extends from the core cladding interface to the penetration depth $d p$, as shown in Fig. 2. The value of $d p$ is derived using Equation (2).

$$
d p=\frac{\lambda}{2 \pi \sqrt{n_{c 1}^{2} \sin ^{2} \theta-n_{e x}^{2}}}
$$

$\theta$ is the incident angle, $\lambda$ is the wavelength, $n_{c l}$ is the cladding refractive index and $n_{e x}$ is the external refractive index (RI of the transformer oil).

As shown in Fig. 2, the value of $d p$ is small and cannot reach the surrounding medium. Thus, the tapering technique is used to allow evanescent fields to penetrate the surrounding medium. In this study, the surrounding medium is transformer oil, and the fibre is tapered using HF as an etchant solution in accordance with the chemical etching method.

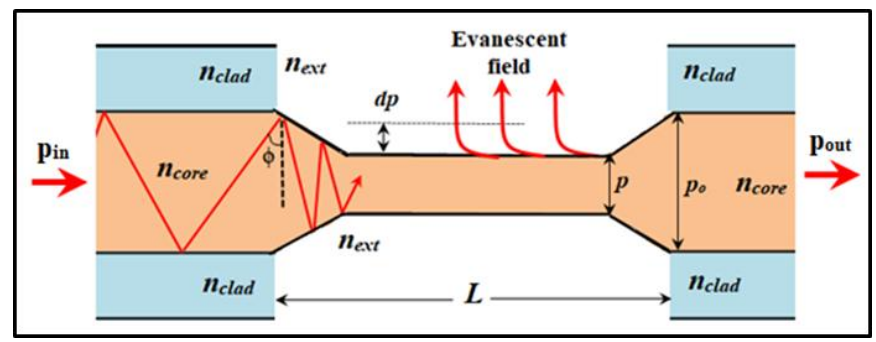

Fig. 2. Cross section of the sensing arm

\section{EXPERIMENTAL PROCEDURE AND MEASUREMENT}

\section{A. Sensor fabrication}

This The fabrication of a bare optical fibre sensor is implemented by chemical etching using $30 \%$ HF. A Plexiglass piece $(100 \mathrm{~mm} \times 50 \mathrm{~mm} \times 10 \mathrm{~mm})$ is used as an etching reactor. The depth of the etching chamber is $5 \mathrm{~mm}$. The Plexiglas material is lightweight and corrosion resistant. The setup for etching process is shown in Fig. 3.

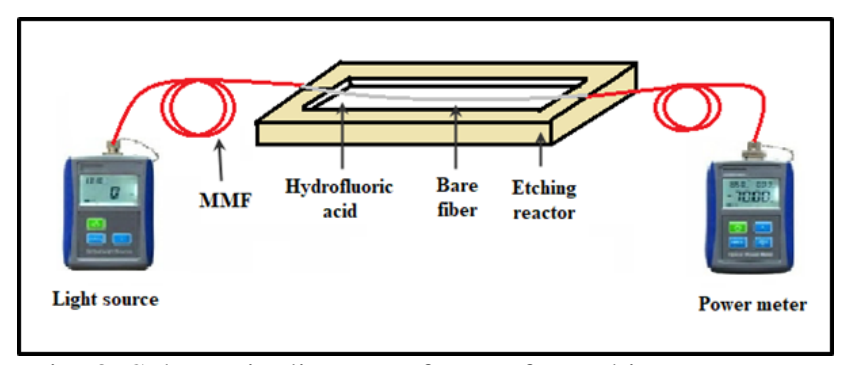

Fig. 3. Schematic diagram of setup for etching process.

The type of fibre used in this experiment is multimode fibre MMF 50/125 $\mu \mathrm{m}$ core/cladding diameter multimode fibre, and the bare length is $8 \mathrm{~cm}$. The cladding is removed from the optical fibre under $60 \mathrm{~min}$ of etching. Throughout the etching process, the sending end of the optical fibre is connected to a $(10 \mathrm{~mW}, 650 \mathrm{~nm})$ laser light source, whereas the receiving end is connected to a power meter (NESTONG T1102). The etching is monitored and controlled on the basis of the light power $(\mathrm{nW})$ measured at the receiving end. The etching is stopped when the output power is approximately $35 \%$ of the total initial power before etching; the removal of the MMF cladding lasts approximately 60 minutes. The characteristics of the output power during etching are shown in Fig. 4. In this figure, the no evanescent field region is starting from (0-24) minute. At this time of etching process, the cladding is not affected by HF, so no evanescent field propagate to fibre wall. The weak evanescent region is started from (24-40) minute. The light starts to propagate to the fibre wall because the fibre cladding is reduced by the effect of HF. At the strong evanescent field (40-60) minute, the HF reached to the core of fibre that is made by glass, so more of lights is propagated to the fibre wall because the core (glass) is highly affected by the HF than cladding. 
At the last stage of etching process, a distilled water is used to wash the fibre from the HF. At this point, the etching process is stopped, and the output power is stable.

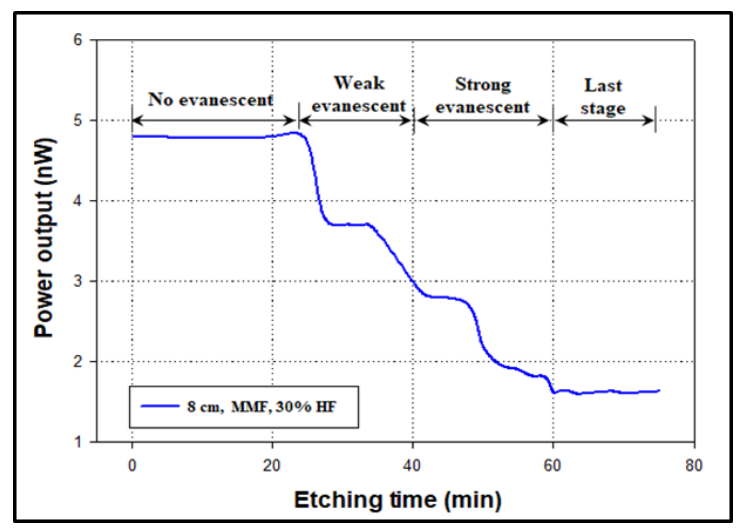

Fig. 4. Optical output power vs. etching time using $30 \% \mathrm{HF}$ solution.

\section{B. Experimental setup and preparation of transformer oil sample}

The bare optical fibre functions as the sensing arm of the MZI configuration. The schematic of the experimental setup using the MZI configuration is shown in Fig. 5 and Table 1 shows the details for each label in the figure. As shown in this figure, the first optical coupler splits the light into two parts (i.e., sensing arm and reference arm). The sensing arm is immersed in transformer oil, whilst the reference arm is not. The second optical coupler then collects the light and sends it to the power meter at the receiving end of the MZI configuration.

In this study, five samples of mineral oil (Libra) are used. $\mathrm{S} 1$ is fresh oil used as a reference, and S2, S3, S4 and S5 are different samples of aged oil prepared by thermal accelerated ageing. In the accelerated ageing process, four types of metal substances $(\mathrm{Cu}, \mathrm{Fe}, \mathrm{Zn}$ and $\mathrm{Al})$ are added into fresh oil according to reference [23]. The resulting mixture is then placed in an oven at $120{ }^{\circ} \mathrm{C}$ for different ageing times. The heat inside the oven increases the ageing rate, as shown in Equations (3), (4) and (5) [24] [25]. If the temperature increases to $7{ }^{\circ} \mathrm{C}$, then the rate of degradation is doubled. The normal operating temperature of transformer oil is $60{ }^{\circ} \mathrm{C}$. Hence, one day of laboratory ageing (inside an oven) is equivalent to a year in the field [1]. Table 2 shows the description of the samples for different ageing times and metal substances.

$$
t_{f}=t_{k} \times 2^{\left.\frac{T_{K}-60}{7}\right)}
$$

Where $t_{f}$ is the equivalent time of ageing, $t_{k}$ is the aging period and $T_{K}$ is the accelerated aging temperature.

$$
\text { Aging accelerating factor }=\frac{120^{\circ} \mathrm{C}-60^{\circ} \mathrm{C}}{7^{\circ} \mathrm{C}}=8.57
$$

Time factor $=2^{8.57}=380$ days

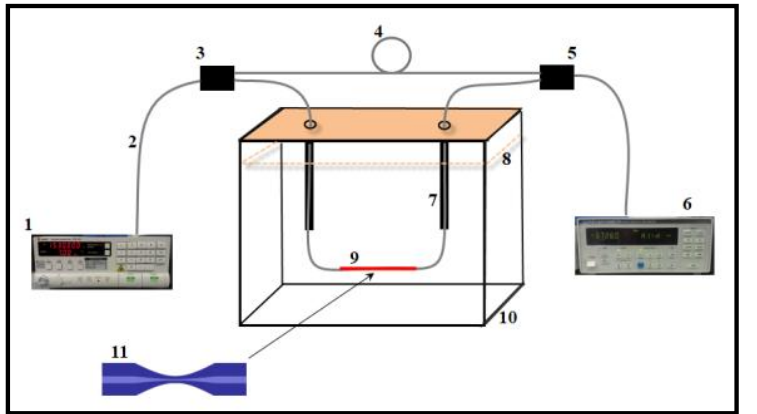

Fig. 5. Schematic of the experimental setup.

Table- I: Details of the test setup

\begin{tabular}{|c|l|}
\hline Label & \multicolumn{1}{|c|}{ Description } \\
\hline 1 & Light source, Santec WSL-100, 1550 nm \\
\hline 2 & $\begin{array}{l}\text { MMF, Core/cladding diameter: } 50.0 / 125 \mu \mathrm{m} \\
\text { RI, Core/cladding: } 1.500 / 1.485\end{array}$ \\
\hline 3,5 & Coupler (1, 2): 50/50 \% \\
\hline 4 & Reference arm, MMF \\
\hline 6 & Optical power meter, ILX Light wave (OMM-6810B) \\
\hline 7 & Holder \\
\hline 8 & Transformer oil level \\
\hline 9 & Sensing arm \\
\hline 10 & Tank \\
\hline 11 & Tapered fiber \\
\hline
\end{tabular}

Table- II: Description of samples

\begin{tabular}{|c|c|c|c|}
\hline Sample name & $\begin{array}{c}\text { Time of } \\
\text { aging }\end{array}$ & $\begin{array}{c}\text { Equivalent } \\
\text { durations in } \\
\text { fields } \\
\text { (Days/Years) }\end{array}$ & \multirow{2}{*}{$\begin{array}{c}\text { Metal } \\
\text { substance }\end{array}$} \\
\hline Sample S1 & 0 hour & $0 / 0$ & $0 \mathrm{~g} / \mathrm{l}$ \\
\hline Sample S2 & 48 hours & $2 / 2$ & $\mathrm{Cu}=2.5 \mathrm{~g} / 1$ \\
\cline { 1 - 2 } Sample S3 & 96 hours & $4 / 4$ & \multirow{2}{*}{$\mathrm{Fe}=2.5 \mathrm{~g} / 1$} \\
\cline { 1 - 2 } Sample S4 & 144 hours & $6 / 6$ & $0.5 \mathrm{~g} / 1$ \\
\cline { 1 - 2 } Sample S5 & 192 hours & $8 / 8$ & $\mathrm{Al}=0.5 \mathrm{~g} / 1$ \\
\hline
\end{tabular}

\section{Results and discussion}

Measuring RI depends on the speed of light inside transformer oils. Oil contamination decreases the speed of light propagating inside transformer oils. Figure 6 illustrates the RI of the transformer oil samples measured at $24{ }^{\circ} \mathrm{C}$ using a digital refractometer (DR102). The RI of this device ranges from 1.3330 to 1.5177. As shown in Fig. 6, the RI of fresh oil is 1.4611 , while that of aged oil is 1.4722 . Hence, as the time of ageing increases, the RI increases.

A breakdown voltage test is conducted on the five samples according to the standard ASTM D1816-84a [26]. The high ageing transformer oil has low breakdown voltage and high $\mathrm{RI}$; the opposite is true for the low ageing samples. 
The moisture and chemical decomposition of the conducting material increase due to the temperature and ageing process. As such, the internal characteristics of the transformer oil changes, thereby reducing the quality of the oil and the value of the breakdown voltage. Fig. 7 illustrates the characteristics of the RI and breakdown versus time of ageing.

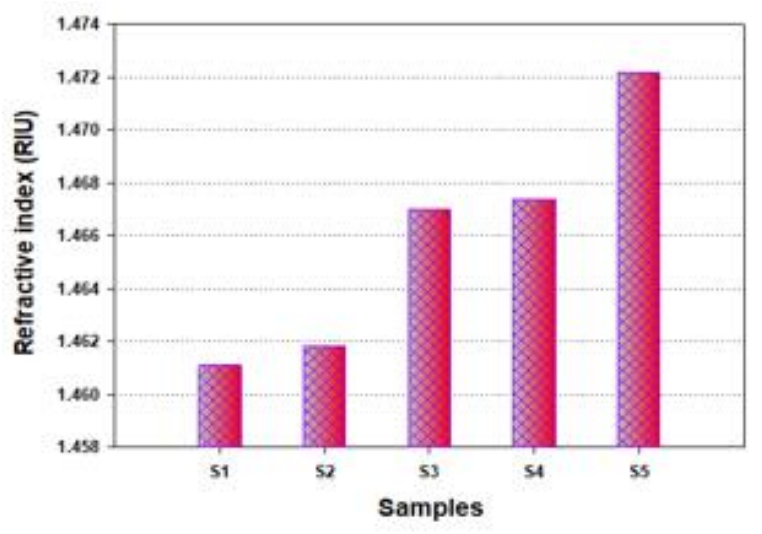

Fig. 6. Refractive index of oil samples at $24{ }^{\circ} \mathrm{C}$.

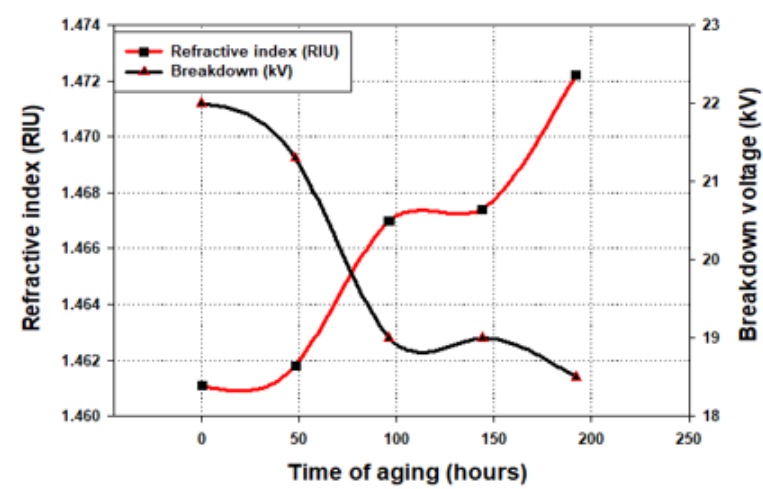

Figure 7: Refractive index and breakdown vs. time of ageing

A laser Santec WSL-100 light source projects light to the sending end of the MZI configuration. The light is divided into two parts by the first optical coupler 50/50\%. This coupler sends the light to the sensing and reference arms. The sensing arm is a tapered MMF fibre immersed in transformer oil, as shown in Figure 5. The second coupler functions as a summation device that collects the light from the reference and sensing arms and then sends the light to the receiving end of the MZI configuration. At the receiving end, the power intensity of the light is measured by using an ILX light wave power meter. Fig. 8 illustrates the characteristics of the output power of the MZI sensor and the value of the breakdown voltage for all samples.

The RI of the transformer oil changes because of different thermal ageing times. A low-quality transformer oil has a high RI, whereas a high-quality transformer oil has a low RI. At the sensing arm of the MZI configuration, the light leaks out to the surrounding medium (i.e., transformer oil) as the evanescent field. The quantity of the light that leaks out depends on the RI of the transformer oil. Thus, we obtain at the receiving end a high output power for a low-quality transformer oil and a low output power for fresh oil.

The absorption spectrum signal of the transformer oil is measured from $355 \mathrm{~nm}$ to $405 \mathrm{~nm}$ by using a UV-visible spectrophotometer (SHIMADZU, UV-1800), as shown in
Fig. 9. In this figure, the low-quality transformer oil has a higher absorption spectrum than the high-quality oil does. The characteristics of the absorption spectrum differ for various oil samples because the physical properties of transformer oils are different and depend on the thermal ageing time.

From above, it seems the correlation is clear between the oil breakdown test, refractive index and absorption spectrum test. When the oil is degrade, the value of breakdown voltage will decrease and at the same time the RI increases. On the other hand, the oil absorption spectrum will be increased. So, the MZI sensor can detect the ageing of transformer oil based on different output power.

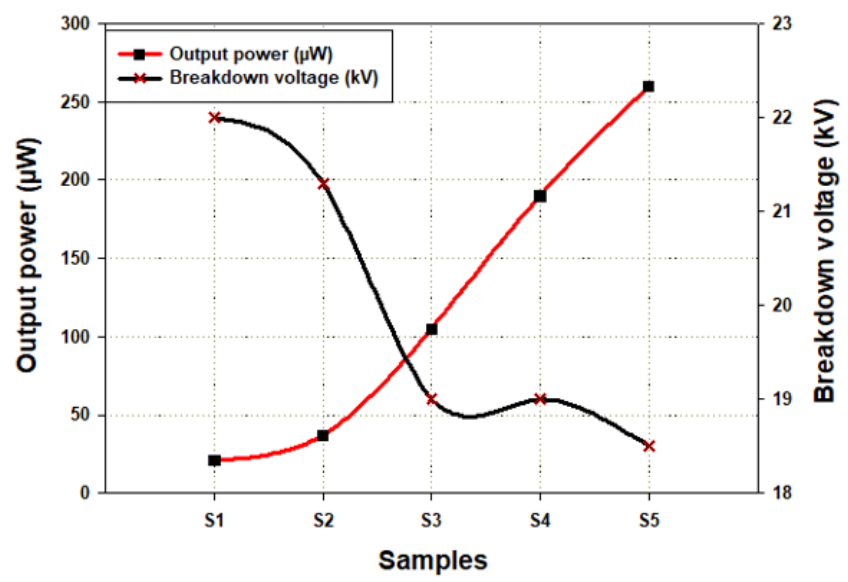

Fig. 8. Characteristics of output power and breakdown voltage.

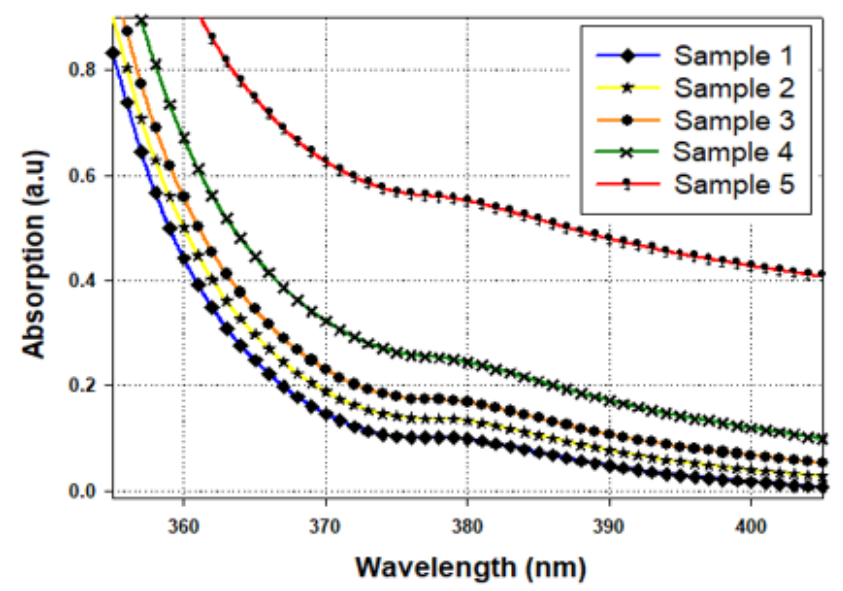

Fig. 9 Absorption spectrum signal of transformer oil samples

\section{CONCLUSION}

A fibre optic sensor based on MZI configuration was developed with a bare fibre as the sensing arm of the sensor. The fibre cladding was removed by chemical etching using $\mathrm{HF}$ as an etchant. The sensor was affected by the RI of the transformer oils. Moreover, it could detect the ageing of transformer oils on the basis of the changes in its RI. As a result, the output power of the MZI sensor was affected by the quality of the transformer oil (i.e., transformer oil RI).

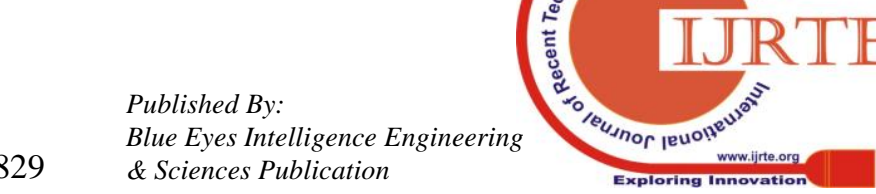


The results showed that the output power is in line with the breakdown voltage test. The sensor output has correlation with the oil absorption spectrum test. This study is important in improving the online monitoring of high voltage transformers as it provides early information about the quality of transformer oils and thus increases the reliability of power system networks.

\section{ACKNOWLEDGMENT}

The authors wish to thank the Ministry of Education Malaysia, Universiti Teknikal Malaysia Melaka (UTeM) and Ministry of Electricity in Iraq for the encouragement. This work is partially funded under Fundamental Research Grant Scheme (FRGS/1/2016/TK04/FKE-CERIA/F00304).

\section{REFERENCES}

1. G M. Kohtoh, G. Ueta, S. Okabe, and T. Amimoto, "Transformer insulating oil characteristic changes observed using accelerated degradation in consideration of field transformer conditions," IEEE Transactions on Dielectrics and Electrical Insulation, vol. 17, no. 3, pp. 808-818, 2010.

2. M. A. Salam, Q. M. Rahman, F. Wen, S. P. Ang, and W. Voon, "Causes of transformer failures and diagnostic methods - A review," Renewable and Sustainable Energy Reviews, vol. 82. pp. 1442-1456, 2018.

3. M. Wang, A. J. Vandermaar, and K. D. Srivastava, "Review of condition assessment of power transformers in service," IEEE Electrical insulation magazine, vol. 18, no. 6, pp. 12-25, 2002.

4. M. Bagheri, M. S. Naderi, T. Blackburn, and B. T. Phung, "Dean-Stark vs FDS and KFT methods in moisture content recognition of transformers," In Power and Energy (PECon), 2012 IEEE International Conference, no. December, pp. 712-717, 2012.

5. I. Hurezeanu, C. I. Nicola, D. Sacerdotianu, M. Nicola, A. M. Aciu, and M. C. Nitu, "Temperature control and monitoring system for power transformer windings using fiber optic sensors," Fundamentals of Electrical Engineering (ISFEE), 2016 International Symposium on. IEEE, 2016., pp. 1-4, 2016.

6. S. Ab Ghani, N. A. Muhamad, I. S. Chairul, and N. Jamri, "A study of moisture effects on the breakdown voltage and spectral characteristics of mineral and palm oil-based insulation oils," ARPN J. Eng. Appl. Sci., vol. 11, no. 8, pp. 5012-5020, 2016.

7. A. B. Lobo Ribeiro, N. F. Eira, J. M. Sousa, P. T. Guerreiro, and J. R. Salcedo, "Multipoint fiber-optic hot-spot sensing network integrated into high power transformer for continuous monitoring," IEEE Sensors Journal, vol. 8, no. 7, pp. 1264-1267, 2008.

8. S. Karmakar, N. K. Roy, and P. Kumbhakar, "Effect of ageing in transformer oil using UV-visible spectrophotometeric technique," Journal of Optics, vol. 40, no. 2, pp. 33-38, 2011.

9. P. Zubiate, J. M. Corres, C. R. Zamarreno, I. R. Matias, and F. J. Arregui, "Fabrication of Optical Fiber Sensors for Measuring Ageing Transformer Oil in Wavelength," IEEE Sensors Journal, vol. 16, no. 12, pp. 4798-4802, 2016.

10. L. Z. Maria, Letizia , Nunzio Cennamo, "Optical chemical sensor for oil-filled power transformer Nunzio," Photonics Technologies, 2014 Fotonica AEIT Italian Conference, vol. 12, pp. 1-3, 2014.

11. N. A. Bakar and A. Abu-Siada, "A new method to detect dissolved gases in transformer oil using NIR-IR spectroscopy," IEEE Transactions on Dielectrics and Electrical Insulation, vol. 24, no. 1, pp. 409-419, 2017.

12. N. Abu Bakar, A. Abu-Siada, N. Das, and S. Islam, "Effect of Conducting Materials on UV-Vis Spectral Response Characteristics," Universal Journal of Electrical and Electronic Engineering, vol. 1, no. 3, pp. 81-86, 2013.

13. S. Laskar and S. Bordoloi, "Monitoring of Moisture in Transformer Oil Using Optical Fiber as Sensor," Journal of photonics, vol. 2013, pp. 1-7, 2013.

14. D. K. Mahanta and S. Laskar, "Water quantity based quality measurement of transformer oil using polymer optical fiber as sensor," IEEE Sensors Journal, vol. 1748, no. c, 2017

15. B. Sarkar, D. K. Mishra, C. Koley, N. K. Roy, and P. Biswas, "Intensity Modulated Fiber Bragg Grating Sensor for Detection of Partial Discharges inside High Voltage Apparatus," IEEE Sensors Journal, no. c, pp. 1-8, 2016.
16. J. Harris, P. Lu, H. Larocque, L. Chen, and X. Bao, "In-fiber Mach-Zehnder interferometric refractive index sensors with guided and leaky modes," Sensors and Actuators B: Chemical, vol. 206, pp. 246-251, 2015.

17. B. H. Lee et al., "Interferometric fiber optic sensors," Sensors, vol. 12, no. 3, pp. 2467-2486, 2012.

18. H. Wang et al., "Simultaneous measurement of refractive index and temperature based on asymmetric structures modal interference," Optics Communications, vol. 364, pp. 191-194, 2016.

19. Y. Sun et al., "Dual-parameters optical fiber sensor with enhanced resolution using twisted MMF based on SMS structure," IEEE Sensors Journal, vol. 17, no. 10, pp. 3045-3051, 2017.

20. A. A. Jasim et al., "Refractive index and strain sensing using inline Mach-Zehnder interferometer comprising perfluorinated graded-index plastic optical fiber," Sensors and Actuators A: Physical, vol. 219, pp. 94-99, 2014.

21. C. X. Teng, F. Da Yu, N. Jing, and J. Zheng, "The influence of temperature to a refractive index sensor based on a macro-bending tapered plastic optical fiber," Optical Fiber Technology, vol. 31, pp. 32-35, 2016.

22. R. T. Ghahrizjani, H. Sadeghi, and A. Mazaheri, "A Novel Method for onLine Monitoring Engine Oil Quality Based on Tapered Optical Fiber Sensor," IEEE Sensors Journal, vol. 16, no. 10, pp. 3551-3555, 2016.

23. S. Tenbohlen and M. Koch, "Aging Performance and Moisture Solubility of Vegetable Oils for Power Transformers," IEEE Transactions on Power Delivery, vol. 25, no. 2, pp. 825-830, 2010.

24. M. M. Emara, D. E. A. Mansour, and A. M. Azmy, "Dielectric properties of aged mineral oil filled with $\mathrm{TiO} 2$ nanoparticles," Electric Power and Energy Conversion Systems (EPECS), 2015 4th International Conference, pp. 15-19, 2015.

25. A. M. Alshehawy and D. A. Mansour, "Impact of Thermal Aging of Transformer Oil on UV-Vis Optical Spectrum and Dielectric Properties," Power Systems Conference (MEPCON), 2016 Eighteenth International Middle East. IEEE, pp. 860-865, 2016.

26. ASTM D1816-03, "Standard Test Method for Dielectric Breakdown Voltage of Insulating Liquids Using VDE Electrodes ,” pp. 1-5, 2014.

\section{AUTHORS PROFILE}

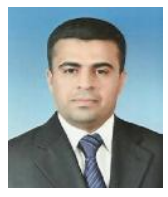

Alaa Razzaq B.Sc. in Electrical Engineering University of Technology Iraq, M.Sc. in Electrical-power, University of Technology Malaysia (UTM), currently he is $\mathrm{PhD}$ Student in Universiti Teknikal Malaysia Melaka (UTeM), his research interests are high voltage transformer assesment and optical fibre sensors.

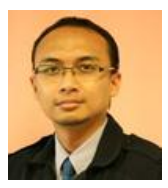

Hidayat Zainuddin Ph.D. in Electronic and Electrical Engineering (High Voltage), University of Southampton, UK, M.Sc. in Electrical Power Engineering with Business, University of Strathclyde, UK, B.Eng. (Electrical), UTM, his research interests are Condition monitoring of high voltage equipment, testing and failure/degradation analysis of insulation/dielectric materials and finite element analysis modelling.

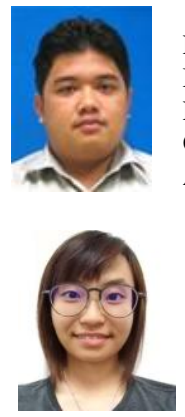

Farhan Hanaffi Ph.D in Electronic and Electrical Engineering, University of Strathclyde, United Kingdom M.Eng in Electrical (UTM), B. Eng (Hons) in Electrical Engineering (UTM), his research interests are Electrical Grounding, Lightning and transient analysis, Finite Element Analysis Modelling

Tan Ying She received the B.Sc. degree in electrical engineering from Universiti Teknikal Malaysia Melaka (UTeM), her research interests are ageing of transformer oil insulation and optical fibre sensors. 
Radhi M. Chyad He received the B.Sc. degree from departure of Physics, Science College- Basra University, Iraq in 1985. He received M.Sc. in laser technology from Technology University- Baghdad, Iraq in 1990 and his $\mathrm{Ph} . \mathrm{D}$. in programs field in engineering applied physics, photonics devises and nano optics technology, from USM, Malaysia, currently worked in Laser \& Optoelectronics Research Center, Directorate of Materials Research, Ministry of Science \& Technology, Baghdad, IRAQ.

Hanim Abdul Razak PhD (Micro and Nanoelectronic Engineering) from UKM, M.Sc (Microelectronics) from UKM, B.Eng (Computer and Information) from UIAM, her research area(s) Nanoelectronics, silicon photonics, device modelling and design, optimization approach (DOE), currently she is Senior Lecturer in Universiti Teknikal Malaysia Melaka (UTeM). 